\title{
Abstract B69: The effects of patient-physician relationships on perceptions of breast cancer treatment in African American women
}

Alexandria N. Lauray and Silvia Bigatti

2018

\begin{abstract}
According to Indiana.gov, 4,400 new cases of breast cancer are diagnosed in Indiana annually. "During 2008 to 2012, the mortality rates of African American breast cancer patients were almost $40 \%$ higher than Caucasian Americans" (Cancer.org), which sheds light on the health disparities African Americans face in the United States. Disparities among cancer patients have not only led to African American women (AAW) being diagnosed in later stages of this disease than their counterparts and thus raising their mortality rates, but AAW tend to be diagnosed with more aggressive forms of cancer such as triplenegative breast cancer, which occurs in $10 \%$ to $20 \%$ of patients. AAW face many barriers today: limited access to screening services, lack of quality screening equipment, limited access to treatment services, and an unspoken distrust of health care providers. Research indicates that during their breast cancer treatment trajectory, AAW experience delay in initiating chemotherapy after surgery, less satisfaction with treatment, increased symptoms, and lower participation in clinical trials (Mcarthy). Despite this, there is no study that has followed and assessed AAW during the treatment sessions to examine challenges in their lives and their impact. Studies of AAW who have survived breast cancer suggest the need to look not only at factors within the medical care system, but well beyond it into the everyday lives of these women and the resources available to them through social networks and other means to overcome challenges. This project explores themes in the baseline and exit interviews of 38 participants. In their initial interview, we asked questions related to support system, their views of treatment going into the process, and the quality of care that they feel they have received. At the end of treatments, we ask about patient-physician relationships and how the women perceived their care. Women in the study reported the need to readdress concerns with physicians. Patient-physician relationships among AAW diagnosed with breast cancer have been strained and have had a negative impact on patient satisfaction with care.
\end{abstract}

Lauray, A. N., \& Bigatti, S. (2018). Abstract B69: The effects of patient-physician relationships on perceptions of breast cancer treatment in African American women. Molecular Cancer Research, 16(8 Supplement), B69-B69. https://doi.org/10.1158/1557-3125.ADVBC17-B69 
Lauray, A. N., \& Bigatti, S. (2018). Abstract B69: The effects of patient-physician relationships on perceptions of breast cancer treatment in African American women. Molecular Cancer Research, 16(8 Supplement), B69-B69. https://doi.org/10.1158/1557-3125.ADVBC17-B69 